\title{
Medium Modification of Vector Mesons
}

\section{Chaden Djalali ${ }^{1}$}

University of South Carolina

Department of Physics and Astronomy, Columbia, SC 29208, USA

E-mail: djalaliesc.edu

\section{Michael Paolone}

University of South Carolina

Department of Physics and Astronomy, Columbia, SC 29208, USA

E-mail: mpalone@jlab.org

\section{Dennis Weygand}

Thomas Jefferson National Accelerator Facility

Newport News, VA 23606, USA

E-mail: weygandejlab.org

\section{Michael H. Wood}

Canisius College

Department of Physics, Buffalo, NY 14208, USA

E-mail: wood5@canisius.edu

\section{Rakhsha Nasseripour}

The George Washington University

Department of Physics, Washington, DC 20052, USA

E-mail: rakhsha@jlab.org

\begin{abstract}
The theory of the strong interaction, Quantum Chromodynamics (QCD), has been remarkably successful in describing high-energy and short-distance-scale experiments involving quarks and gluons. However, applying QCD to low energy and large-distance scale experiments has been a major challenge. Various QCD-inspired models predict a partial restoration of chiral symmetry in nuclear matter with modifications of the properties of hadrons from their free-space values. Measurable changes such as a shift in mass and/or a change of width are predicted at normal nuclear density. Photoproduction of vector mesons off nuclei have been performed at different laboratories. The properties of the $\rho, \omega$ and $\varphi$ mesons are investigated either directly by measuring their mass spectra or indirectly through transparency ratios. The latest results regarding medium modifications of the vector mesons in the nuclear medium will be discussed.
\end{abstract}

35th International Conference of High Energy Physics (ICHEP2010)

Paris,France

July 22-28, 2010

1 Speaker 


\section{Introduction}

The possible modification of the properties of vector mesons, such as a shift in their masses and/or broadening of their widths in dense nuclear matter has generated tremendous interest both experimentally and theoretically. Several comprehensive reviews have been written about these medium modifications. ${ }^{1,2}$ These modifications can be related to partial restoration of chiral symmetry in the nuclear medium.

\section{Vector mesons in Nuclei $(T=0$ and $\rho \sim \rho 0)$}

Many experiments have been studying the properties of the $\rho, \omega$ and $\phi$ mesons in the nuclear medium, using simple probes such as $\gamma, \pi$ or $p$ on nuclei ( $T=0$ and $\rho \sim \rho_{0}$ ). The $\rho$ meson with its very short lifetime has a large probability of decaying in the medium. It is the best candidate for observing any medium modification by measuring its in medium mass spectrum. The $\omega$ and $\varphi$ mesons mostly decay outside the medium in which they are produced making it very difficult to directly measure any medium modification. Their in medium widths can however be determine through transparency ratios. The most interesting property of these vector mesons is that they decay into di-leptons, which have negligible final state interactions. However, measuring the di-lepton spectra is very challenging because of the very small branching ratio $\left(\sim 10^{-5}\right)$.

\subsection{Direct measurements through invariant mass spectra}

The TAGX collaboration was one of the first experiments to report medium modification of the $\rho$-meson. It reported a large decrease of the $\rho$-meson mass $(\sim 20 \%)$ in the reaction ${ }^{3} \mathrm{He}\left(\gamma, \pi^{+} \pi^{-}\right) \mathrm{X}$, where the pion pairs result from sub-threshold $\rho$-meson production and decay. ${ }^{3} \mathrm{~A}$ later analysis of the data based on the longitudinal polarization of the $\rho$ mesons found a smaller but significant decrease in mass. ${ }^{4}$ However these results are surprising given the small density of the nucleus and the final-state interactions on the pion pairs. The E325 experiment at the KEK Proton Synchrotron was the first to search for the modification of the vector mesons in the nucleus in the di-lepton decay channel. They measured the invariant mass spectra of $\mathrm{e}^{+} \mathrm{e}^{-}$pairs produced in $12 \mathrm{GeV}$ proton-induced nuclear reactions. The background subtracted invariant mass spectra measured on $\mathrm{Cu}$ seem to indicate a downward mass shift of $9.2 \pm 0.2 \%$ for the $\rho$ meson and no change in the widths of the $\rho$ and $\omega$ mesons. ${ }^{5,6}$ The analysis of the $\varphi$ meson shows a downward mass shift of $3.4 \pm 0.6 \%$ of its mass and an increase of a factor 3-4 of its width. The CBELSA/TAPS collaboration at the electron stretcher accelerator (ELSA) in Bonn used the $\gamma \mathrm{A} \rightarrow \pi^{0} \gamma \mathrm{X}$ reaction to study the $\omega$ meson in-medium behavior in $\mathrm{Nb}$. The branching ratio for $\omega$ $\rightarrow \pi^{0} \gamma$ decay is the order of $9 \%$. This channel provides a clean and exclusive mode to study the $\omega$ in-medium properties since the $\rho$ meson is highly suppressed in this decay channel. However, a disadvantage is the strong final state interactions of the $\pi^{0}$ meson in the nucleus. In a first analysis, a shoulder on the low-mass side of the $\omega$ peak was found in the $\mathrm{Nb}$ target and was interpreted as medium modified $\omega$-mesons with a downward mass shift of $14 \%{ }^{7}$ In a reanalysis of the data, the shoulder has disappeared and the results are compatible with no mass shift. ${ }^{8}$ 
The E01-112 (g7) experiment was conducted in Hall B at JLab using the Cebaf Large Acceptance Spectrometer (CLAS). The vector mesons were photo-produced on $\mathrm{LD}_{2}, \mathrm{C}, \mathrm{Fe}, \mathrm{Ti}$ and $\mathrm{Pb}$. The outgoing vector mesons were reconstructed from their decay to $\mathrm{e}^{+} \mathrm{e}^{-}$pairs. The CLAS detector is ideal for this experiment owing to its ability to detect multi-particle final states and its high e $/ \pi$ rejection factor. Effective discrimination between lepton and pion pairs to the level of $10^{-7}$ is easily achieved. The same-charge pairs method was successfully used to determine the combinatorial background. Once the combinatorial background subtracted, the mass spectra mainly consist of the $\rho, \omega$ and $\varphi$ mesons. The invariant mass spectra are fitted using spectral shapes calculated by the semi-classical BUU transport model developed by the Giessen group. ${ }^{9}$ The fits describe the data very well and are consistent with the PDG values (no mass shift) and the widths are consistent with the collisional broadening $(\Delta \Gamma \sim+70 \mathrm{MeV}$ in $\mathrm{Fe}){ }^{10,11}$

\subsection{Transparency ratios}

The $\omega$ and $\varphi$ mesons, due to their longer lifetimes, mostly decay outside of the nucleus. The information on the in-medium width of these mesons can be extracted from their interactions with the nucleons as the mesons escape the nucleus using the transparency ratio defined (for a vector meson V) as $\boldsymbol{T}_{A}=\sigma_{\gamma A \rightarrow V X} / \boldsymbol{A} . \sigma_{\gamma N \rightarrow V X}$ where $\sigma_{\gamma N \rightarrow V X}$ is the inclusive nuclear vector-meson photo-production cross section and $\sigma \gamma \mathrm{N} \rightarrow \mathrm{VX}$ is cross section on a free nucleon. The transparency ratio $\boldsymbol{T}_{\boldsymbol{A}}$ is a measure of the loss of vector-meson flux via inelastic processes in the nucleus, and is related to the absorptive part of the meson-nucleus potential. Extracting the in-medium meson width from the A dependence of the ratio $\boldsymbol{T}_{\boldsymbol{A}}$ requires comparison with theoretical calculations. ${ }^{12,13}$ For the $\omega$-meson measured in the JLab g7 experiment, the transparency ratios are derived for the ${ }^{2} \mathrm{H}, \mathrm{C}, \mathrm{Fe} / \mathrm{Ti}$, and $\mathrm{Pb}$ and compared to the CBELSA/TAPS data ${ }^{14}$ and theoretical calculations. The transparency ratios decrease rapidly as a function of A, indicating a substantial increase in the in-medium width of the $\omega$. The JLab ratios drop faster that the CBELSA-TAPS ratios indicating an even larger in-medium width $\left(\Gamma_{\omega}\right.$ $\geq 200 \mathrm{MeV}$ ). The CBELSA/TAPS collaboration has published a value of $\Gamma_{\omega} \sim 130-150 \mathrm{MeV}$. The very large $\omega$-meson absorption observed in the $\mathrm{e}^{+} \mathrm{e}^{-}$channel cannot be explained with the current theoretical calculations. ${ }^{15,16}$ The photo-production of $\varphi$ mesons from $\mathrm{Li}, \mathrm{C}, \mathrm{Al}$ and $\mathrm{Cu}$ targets has been measured at $\mathrm{E}_{\gamma}=1.5-2.4 \mathrm{GeV}$ at SPring-8 (LEPS), in the $\gamma \mathrm{A} \rightarrow \mathrm{K}^{+} \mathrm{K}^{-} \mathrm{X}$ channel. ${ }^{17}$ Using a Glauber-type model calculation, the in-medium $\varphi$-nucleon cross section was deduced to be $\sigma_{\varphi \mathrm{N}}=37[+17,-11] \mathrm{mb}$, which is much larger than the free-space value used as an input in most model calculations. The SPring8 results are compatible within statistical uncertainties with the $\mathrm{JLab}_{\text {results }}{ }^{18}$ and seem to indicate an in-medium width for the $\varphi$ as large as $\Gamma_{\varphi} \sim 70 \mathrm{MeV}$.

\section{Summary and Conclusions}

The in-medium properties of the light vector mesons have been studied in several nuclei by different elementary reactions. The recent photoproduction experiments do not observe any mass shift but a substantial broadening of the $\rho$-meson consistent with collisional broadening in the medium. These results are consistent with the observations reported by heavy ion 
experiments. The $\omega$ and $\varphi$ meson widths deduced from transparency-ratio measurements are factors of 5 to 15 times larger than in the vacuum. The latest models predict that most of the medium modifications happen when the meson have momenta less than $0.7 \mathrm{GeV} / \mathrm{c}$ with respect to the medium. Next generation experiments with much higher statistics and improved acceptance for low momenta mesons are needed to obtain reliable results. Several experiments are planned at JPARC, JLab, RHIC, FAIR and CERN. During the next decade, substantial theoretical and experimental efforts will continue to be carried out in this very active field.

\section{References}

[1] R.S. Hayano and T. Hatsuda, (2009) arXiv:nucl-ex/0812.1702v2.

[2] S. Leupold, V. Metag and U. Mosel, (2009) arXiv:nucl-th/0907.2388v1.

[3] G. J. Lolos et al., Phys. Rev. Lett. 80 (1998) 241.

[4] G. M. Huber et al., Phys. Rev. C 68 (2003) 065202.

[5] R. Muto et al., Phys. Rev. Lett. 98 (2007) 042501.

[6] M Naruki et al., Phys. Rev. Lett. 96 (2006) 092301.

[7] D. Trnka et al., Phys. Rev. Lett. 94 (2005) 192303.

[8] M. Nanova et al., Phys. Rev. C 82, 035209 (2010).

[9] P Muehlich et al., (2002) arXiv:nucl-th/0210079.

[10] R. Nasseripour et al., Phys. Rev. Lett. 99 (2007) 262302.

[11] M. H. Wood et al., Phys. Rev. C 78 (2008) 015201.

[12] M. Kaskulov et al., Phys. Rev. C 75 (2007) 064616.

[13] P. Muehlich and U. Mosel, Nucl. Phys. A 765 (2006) 188;

D. Cabrera et al., Nucl. Phys. A 733 (2004) 130.

[14] M. Kotulla et al., Phys. Rev. Lett. 100 (2008) 192302.

[15] P. Muehlich and U. Mosel, Nucl. Phys. A 773 (2006) 156.

[16] M. Kaskulov et al., Eur. Phys. J. A 31 (2007) 245.

[17] T. Ishikawa et al., Phys. Lett. B 608 (2005) 215.

[18] M. H. Wood et al., Phys. Rev. Lett. 105 (2010) 112301. 